\title{
Phase transitions in $q$-deformed 2D Yang-Mills theory and topological strings
}

\author{
Xerxes Arsiwalla, ${ }^{1}$ Rutger Boels, ${ }^{1}$ Marcos Mariño, ${ }^{2, *}$ and Annamaria Sinkovics ${ }^{1}$ \\ ${ }^{1}$ Institute for Theoretical Physics, University of Amsterdam, Valckenierstraat 65, 1018 XE Amsterdam, The Netherlands \\ ${ }^{2}$ Department of Physics, Theory Division, CERN, CH-1211 Geneva, Switzerland
}

(Received 12 October 2005; published 25 January 2006)

\begin{abstract}
We analyze large $N$ phase transitions for $U(N) q$-deformed two-dimensional Yang-Mills theory on the sphere. We determine the phase diagram of the model and we show that, for small values of the deformation parameter, the theory exhibits a phase transition which is smoothly connected to the DouglasKazakov phase transition. For large values of the deformation parameter the phase transition is absent. By explicitly computing the one-instanton suppression factor in the weakly coupled phase, we also show that the transition is triggered by instanton effects. Finally, we present the solution of the model in the strongly coupled phase. Our analysis suggests that, on certain backgrounds, nonperturbative topological string theory has new phase transitions at small radius. From the point of view of gauge theory, it suggests a mechanism to smooth out large $N$ phase transitions.
\end{abstract}

DOI: 10.1103/PhysRevD.73.026005

PACS numbers: $11.25 . \mathrm{Sq}, 11.15 . \mathrm{Pg}$

\section{INTRODUCTION}

In certain cases, nonperturbative completions of string theory can be obtained by considering a holographic description in terms of a D-brane gauge theory. Recently, in the case of topological strings, Ooguri, Strominger, and Vafa [1] made a proposal for such a nonperturbative completion based on the connection to the black hole attractor mechanism. According to [1], the nonperturbative description of topological string theory on a Calabi-Yau background is encoded in a D-brane gauge theory living on some appropriate cycles of the manifold.

In $[2,3]$ this proposal was made more concrete by considering Calabi-Yau backgrounds of the form

$$
L_{1} \oplus L_{2} \rightarrow \Sigma_{g},
$$

where $\Sigma_{g}$ is a Riemann surface of genus $g$ and $L_{1}, L_{2}$ are line bundles such that $\operatorname{deg}\left(L_{1}\right)+\operatorname{deg}\left(L_{2}\right)=2 g-2$. In this case, the relevant D-brane gauge theory reduces to a $q$-deformed version of two-dimensional Yang-Mills (YM) theory on the Riemann surface $\Sigma_{g} . q$-deformed 2D YM can be regarded as a one-parameter deformation of the standard 2D YM theory. As we will explain below, the deformation can be parametrized by a real, positive number $p$, in such a way that as $p \rightarrow \infty$ one recovers the standard YM theory. The $q$-deformed theory is exactly solvable and one can compute its partition function on any Riemann surface. This partition function has a strong coupling expansion as a sum over representations of the gauge group, which can be written, following [4], in terms of a product of a chiral and an antichiral sector. The perturbative topological string partition function, which was computed in [5] for this class of geometries, is given by a certain limit of this expansion in which the antichiral sector decouples. Once we have a

*Also at Departamento de Matemática, IST, Lisboa, Portugal. nonperturbative description of the theory, it is natural to ask what new phenomena emerge in this description and what their implications are for string theory. For example, in [6] the fermionic description of 2D YM on the torus was used to study baby universes in string theory.

2D YM theory on the sphere exhibits an interesting phenomenon: as shown by Douglas and Kazakov [7], there is a large $N$, third order phase transition at a critical value of the area $A=\pi^{2}$ between a large area phase and a small area phase. From the point of view of the small area/weak coupling phase, the phase transition is triggered by instantons [8]. From the point of view of the large area/strong coupling phase and its string description in terms of branched coverings [4,9], the transition is triggered by the entropy of branch-point singularities [10]. Because of the Douglas-Kazakov transition, the large area expansion of 2D YM theory on the sphere has a finite radius of convergence [10].

In this paper we will study the possibility of large $N$ phase transitions in $q$-deformed 2D YM. Since as the deformation parameter $p$ goes to infinity we recover the usual theory, it is natural to expect the transition to occur at large enough values of $p$. In fact, our result show that the transition persists for all $p>2$, and we find a critical line smoothly connected to the Douglas-Kazakov transition of the standard 2D YM theory. We also show that for $p \leq 2$, in the regime of strong $q$-deformation, the phase transition does not occur. We also perform a detailed instanton analysis which shows that, as in the standard YM case studied in [8], the transition is triggered by instanton effects.

Most of the analysis of this paper are done in the small area phase. In 2D YM theory this phase is described by a Gaussian matrix model. In the $q$-deformed case, this phase is essentially described by the Chern-Simons or StieltjesWigert matrix model introduced in [11] and studied in 
$[12-14]^{1}$. This model, albeit complicated, is exactly solvable (in terms of, for example, orthogonal polynomials), and this is the underlying reason that we can make exact statements about the location of the critical line and the instanton contributions. The large area phase turns out to be more difficult to handle. In this paper we present some preliminary results and derive the equations that determine the full solution (including an explicit expression for the two-cut resolvent). We expect the phase transition of the $q$-deformed theory to be of third order for $p>2$, since it is smoothly connected to the transition of Douglas and Kazakov, and indeed we give indirect evidence that this is so.

As in the standard 2D YM, the existence of the phase transition in the $q$-deformed version indicates that the large area expansion has a finite radius of convergence. According to [2], this theory provides a nonperturbative description of topological string theory on certain CalabiYau backgrounds. This suggests that the large area expansion breaks down in the full topological string theory, and there is a phase transition between a small area phase and a large area phase. From the gauge theory point of view, our analysis shows that when the $q$-deformation is strong enough, the model exhibits a single phase. This suggests that $q$-deformations give a mechanism to smooth out large $N$ phase transitions.

The structure of this paper is as follows: in Sec. II we briefly review the Douglas-Kazakov transition in 2D YM theory. In Sec. III we determine the phase diagram of the $q$-deformed theory and we find a line of critical points parametrized by $p$, for $p>2$. In Sec. IV we adapt the analysis of [8] and study the phase transition of the $q$-deformed theory in terms of instantons in the weakly coupled phase. We find an explicit expression for the oneinstanton suppression factor which indicates that, indeed, the transition is triggered by instanton effects. In Sec. V we analyze the large area phase, which can be encoded by standard techniques in a two-cut solution to an auxiliary matrix model. Finally, in Sec. VI, we discuss the implications of our results for topological string theory and outline some problems opened by this investigation.

As this paper was being completed, we became aware of the work [16], where the same problem is studied. After submission, another paper appeared [17] addressing the same issues.

\section{A REVIEW OF THE DOUGLAS-KAZAKOV TRANSITION}

2D YM theory is an exactly solvable model. In particular, the partition function of the $U(N)$ theory on the sphere is given by a sum over representations of $U(N)$ (see [18]

\footnotetext{
${ }^{1}$ Connections between Chern-Simons theory and $q$-deformed 2D Yang-Mills theory have been made, from a different perspective, in $[2,15]$.
}

and references therein)

$$
Z=\sum_{R}(\operatorname{dim} R)^{2} e^{-A C_{2}(R) / 2 N} e^{i \theta C_{1}(R)},
$$

where $\operatorname{dim} R$ is the dimension of the representation $R, A$ is a real and positive parameter that can be identified with the area of the sphere, and $C_{1}(R), C_{2}(R)$ are the first and second Casimir of $R$. We will represent $R$ by a set of integers $\left\{l_{1}, l_{2}, \cdots, l_{N}\right\}$ satisfying the inequality

$$
\infty \geq l_{1} \geq l_{2} \geq \cdots \geq l_{N} \geq-\infty .
$$

In terms of these integers, the Casimirs have the expression

$$
C_{1}(R)=\sum_{i=1}^{N} l_{i}, \quad C_{2}(R)=\sum_{i=1}^{N} l_{i}\left(l_{i}-2 i+N+1\right) .
$$

Although the above partition function looks rather simple, this theory turns out to have a very rich structure. In $[4,9]$ it was shown that at large area the partition function (2.1) admits a string representation in terms of branched coverings of Riemann surfaces (see [18] for an excellent review). Douglas and Kazakov found that the planar free energy on the sphere exhibits a third order phase transition at the critical value

$$
A_{*}=\pi^{2} .
$$

This large $N$ transition is a continuum analogue of the Gross-Witten-Wadia phase transition for 2D YM theory on the lattice $[19,20]$. Since in this paper we will be considering a generalization of the Douglas-Kazakov phase transition, we will briefly review how this transition is found. For the rest of this section we will set $\theta=0$.

At large $N$ it is natural to introduce a distribution of Young tableaux

$$
n(x)=\frac{l_{i}}{N}, \quad x=\frac{i}{N} .
$$

Defining the shifted distribution

$$
h(x)=-n(x)+x-\frac{1}{2},
$$

one finds that the planar free energy is given by

$$
F_{0}(A)=-S_{G}[h],
$$

where the functional $S_{G}[h]$ reads

$$
\begin{aligned}
S_{G}[h]= & -\int_{0}^{1} d x \int_{0}^{1} d y \log |h(x)-h(y)| \\
& +\frac{A}{2} \int_{0}^{1} d x h(x)^{2}-\frac{A}{24}-\frac{3}{2} .
\end{aligned}
$$

Let us now introduce the density function

$$
\rho(h)=\frac{d x}{d h},
$$


which is normalized to unity,

$$
\int d h \rho(h)=1 \text {. }
$$

One crucial observation of [7] is that, because of the inequality (2.2), this density has to satisfy

$$
\rho(h) \leq 1
$$

for all $h$. We can now write (2.8) as

$$
\begin{aligned}
S_{G}[\rho]= & -\int d h \int d h^{\prime} \rho(h) \rho\left(h^{\prime}\right) \log \left|h-h^{\prime}\right| \\
& +\frac{A}{2} \int d h \rho(h) h^{2}-\frac{A}{24}-\frac{3}{2} .
\end{aligned}
$$

This is (up to the $\rho$-independent terms) the saddle-point functional for a Gaussian matrix model with 't Hooft parameter $t=1 / A$. It then follows that the density $\rho(h)$ is given by Wigner's semicircle law,

$$
\rho_{G}(\lambda, t)=\frac{1}{2 \pi t} \sqrt{4 t-\lambda^{2}}
$$

and we find

$$
\rho(h)=\rho_{G}(h, 1 / A) .
$$

However, it is clear that this solution can be valid only for $A \leq \pi^{2}$, since after this point the inequality (2.11) is violated. This indicates that there is a phase transition at the critical value (2.4).

For $A \geq \pi^{2}$ the Gaussian solution is no longer valid, and Douglas and Kazakov argued that one could obtain a solution for the large area phase by considering a density of eigenvalues of the form,

$$
\begin{cases}\rho(h)=\tilde{\rho}(h), & -a \leq h \leq-b, b \leq h \leq a, \\ 1, & -b \leq h \leq b,\end{cases}
$$

where $b<a$ are points in the real positive axis. From the point of view of the density $\rho(h)$, the Douglas-Kazakov transition can be represented as in Fig. 1: for $A<\pi^{2}$ the Gaussian density gives a good description, but as $A \geq \pi^{2}$ one finds a new density of the form (2.15). It is easy to see that finding $\tilde{\rho}(h)$ amounts to finding a two-cut solution for a modified matrix model with a logarithmic potential. The explicit solution to this problem was worked out in [7], and this allowed them to verify that the phase transition at $A=$
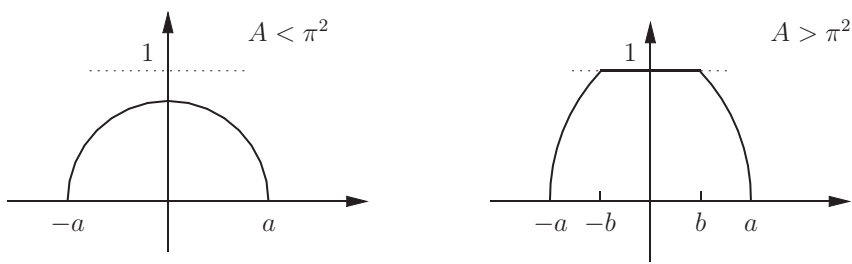

FIG. 1. This figure shows the density $\rho(h)$ before and after the Douglas-Kazakov transition. The solution for $A \geq \pi^{2}$ can be interpreted as a two-cut solution of an auxiliary matrix model. $\pi^{2}$ is of third order. It was also verified that the large area solution agrees with the string expansion of [4].

The mechanism behind the Douglas-Kazakov phase transition was further elucidated in $[8,21,22]$. In particular, it was shown by Gross and Matytsin in [8] that the Douglas-Kazakov phase transition is driven by instantons. The small area phase is dominated by the perturbative vacuum, and instantons are suppressed with an $\exp (-N)$ factor. The one-instanton suppression factor at leading order in $N$ was computed in [8] to be given by

$$
\exp \left[-\frac{N}{A} \gamma_{\mathrm{GM}}(A)\right]
$$

where

$$
\gamma_{\mathrm{GM}}(A)=2 \pi \sqrt{\pi^{2}-A}-A \log \left[\frac{\left(\pi+\sqrt{\pi^{2}-A}\right)^{2}}{A}\right] .
$$

Since $\gamma_{\mathrm{GM}}\left(A=\pi^{2}\right)=0$, as we reach the critical point instantons are not anymore suppressed and they trigger the phase transition, which is then a consequence of $\exp (-N)$ effects which are not visible in the $1 / N$ expansion.

\section{THE PHASE DIAGRAM OF $q$-DEFORMED 2D YM}

The $q$-deformed two-dimensional Yang-Mills theory arises as a natural deformation of the usual model. This model has been considered in $[23,24]$ and more recently, in the context of topological string theory, in [2]. The partition function of the $q$-deformed theory on the sphere can be obtained by replacing the dimensions of representations in (2.1) by their quantum counterpart, in the sense of quantum group theory. The resulting partition function depends on the rank $N$ of the gauge group, two real parameters, $p, g_{s}$, and an angle $\theta$. It reads,

$$
Z^{q}=\sum_{R}\left(\operatorname{dim}_{q} R\right)^{2} q^{p C_{2}(R) / 2} e^{i \theta C_{1}(R)},
$$

where the quantum dimension of $R$ is given by

$$
\operatorname{dim}_{q} R=\prod_{1 \leq i<j \leq N} \frac{\left[l_{i}-l_{j}+j-i\right]}{[j-i]},
$$

and the $q$-numbers appearing here are defined as

$$
[x]=q^{x / 2}-q^{-(x / 2)}, \quad q=e^{-g_{s}} .
$$

The free energy of the model is defined as

$$
F^{q}=\frac{1}{N^{2}} \log Z^{q} .
$$

It is convenient to define the parameter $A$ as

$$
p g_{s}=\frac{A}{N} \text {. }
$$


As we will see in a moment, $A$ corresponds to the area of the sphere in (2.1). As in 2D YM, we will require $A$ to be positive. Notice that the $q$-deformed theory is symmetric under $p, g_{s} \rightarrow-p,-g_{s}$. Therefore, we can restrict ourselves to the range of parameters $p>0, g_{s}>0$.

An important property of the $q$-deformed theory is that in a suitable double-scaling limit, one recovers ordinary 2D YM. This limit is defined as follows:

$$
p \longrightarrow \infty, \quad g_{s} \longrightarrow 0, \quad A, N \quad \text { fixed. }
$$

As $g_{s} \rightarrow 0$ with $N$ fixed, the quantum dimension becomes the classical dimension:

$$
\operatorname{dim}_{q} R \longrightarrow \operatorname{dim} R,
$$

and

$$
q^{p C_{2}(R) / 2} \longrightarrow \exp \left(-\frac{A C_{2}(R)}{2 N}\right)
$$

which is the standard weight factor for 2D YM. We then recover the partition function (2.1) for a sphere of area $A$. The $q$-deformed theory can then be regarded as a oneparameter deformation of 2D YM.

In this paper we will be interested in the large $N$ dynamics of the deformed theory. It is useful to introduce the 't Hooft parameter, which is defined as

$$
t=N g_{s}
$$

and we will consider the 't Hooft large $N$ limit in which $N \rightarrow \infty$ and $t$ and $p$ are fixed. The planar free energy

$$
F_{0}^{q}(t, p)=\lim _{N \rightarrow \infty} F^{q}
$$

will then be a function of $t$ and $p$. Notice that the limit (3.6) that gives ordinary Yang-Mills theory can be implemented order by order in the $1 / N$ expansion by taking

$$
p \longrightarrow \infty, \quad t \longrightarrow 0, \quad p t=A \quad \text { fixed. }
$$

In this way, we recover planar 2D YM on the sphere. We will check many of our results for the $q$-deformed theory, by verifying that in the limit (3.11) one recovers the known results in 2D YM.

In order to compute the planar free energy, we follow the steps outlined in the previous section for the undeformed theory and represent the planar free energy in terms of a functional of a distribution $h(x)$, which is defined as in (2.6). It is easy to see that in the large $N$ limit the planar free energy derived from (3.1) is given by

$$
F_{0}^{q}(t, p)=-S[h],
$$

where the functional $S[h]$ reads

$$
\begin{aligned}
S[h]= & -\int_{0}^{1} d x \int_{0}^{1} d y \log \left|2 \sinh \frac{t}{2}(h(x)-h(y))\right| \\
& +\frac{p t}{2} \int_{0}^{1} d x h(x)^{2}+i \theta \int_{0}^{1} d x h(x)-\frac{p t}{24} \\
& +\int_{0}^{1} d x \int_{0}^{1} d y \log \left|2 \sinh \frac{t}{2}(x-y)\right|
\end{aligned}
$$

and in (3.12) $S[h]$ is evaluated on the configuration $h(x)$ which minimizes the above functional. The last term in (3.13) comes from the denominator of the quantum dimension and it is given by

$$
\int_{0}^{1} d x \int_{0}^{1} d y \log \left|2 \sinh \frac{t}{2}(x-y)\right|=\frac{2}{t^{2}} F_{0}^{\mathrm{CS}}(t),
$$

where

$$
F_{0}^{\mathrm{CS}}(t)=\frac{t^{3}}{12}-\frac{\pi^{2} t}{6}-\mathrm{Li}_{3}\left(e^{-t}\right)+\zeta(3) .
$$

This function is the planar free energy of Chern-Simons theory [25], and we recall that the polylogarithm of order $n$ is given by

$$
\operatorname{Li}_{n}(x)=\sum_{k=1}^{\infty} \frac{x^{k}}{k^{n}}
$$

If we redefine

$$
h(x) \rightarrow h(x)+\frac{i \theta}{t p},
$$

the functional (3.13) becomes

$$
\begin{aligned}
S[h]= & -\int_{0}^{1} d x \int_{0}^{1} d y \log \left|2 \sinh \frac{t}{2}(h(x)-h(y))\right| \\
& +\frac{p t}{2} \int_{0}^{1} d x h(x)^{2}-\frac{p t}{24}+\frac{\theta^{2}}{2 p t}+\frac{2}{t^{2}} F_{0}^{\mathrm{CS}}(t) .
\end{aligned}
$$

Since the inclusion of $\theta$ only leads to an additive term in the planar free energy, we will set $\theta=0$ from now on. After introducing a density function $\rho(h)$ as in (2.10), the $\rho$-dependent part of the effective action can be written (3.13) as

$$
\begin{aligned}
S[\rho]= & -\int d h \int d h^{\prime} \rho(h) \rho\left(h^{\prime}\right) \log \left|2 \sinh \frac{t}{2}\left(h-h^{\prime}\right)\right| \\
& +\frac{p t}{2} \int d h \rho(h) h^{2} .
\end{aligned}
$$

As explained in the previous section, to see if there is a phase transition one first solves for the $\rho(h)$ that extremizes (3.19), assuming a one-cut structure. In order to compute $\rho(h)$, we have to solve the integral equation derived from (3.19),

$$
p h=\mathrm{P} \int d h^{\prime} \rho\left(h^{\prime}\right) \operatorname{coth} \frac{t}{2}\left(h-h^{\prime}\right),
$$

where $\mathrm{P}$ denotes principal value. The density $\rho(h)$ is supported on a symmetric interval $(-a, a)$. A similar in- 
tegral equation appears in the saddle-point analysis of the Chern-Simons matrix model on the three-sphere [11-13]. In fact, after the change of variables $\beta=t h$, (3.19) becomes the planar functional for the Chern-Simons matrix model

$$
Z_{N}=\int \prod_{i=1}^{N} \frac{d \beta_{i}}{2 \pi} \prod_{i<j}\left(2 \sinh \frac{\beta_{i}-\beta_{j}}{2}\right)^{2} \exp \left\{-\frac{N}{2 \xi} \sum_{i=1}^{N} \beta_{i}^{2}\right\},
$$

with 't Hooft parameter $\xi=t / p$. This connection suggests an effective way of solving (3.20). As in [12-14], we change variables

$$
\lambda=\mathrm{e}^{t h+t / p},
$$

and we introduce the density for the new variable $\lambda$,

$$
\rho(\lambda)=\frac{d h}{d \lambda} \rho(h)=\frac{1}{t \lambda} \rho(h) .
$$

The integral Eq. (3.20) becomes

$$
\frac{1}{2} \frac{p}{t} \frac{\log \lambda}{\lambda}=\mathrm{P} \int d \lambda^{\prime} \frac{\rho\left(\lambda^{\prime}\right)}{\lambda-\lambda^{\prime}} .
$$

This is exactly the saddle-point equation for the ChernSimons/Stieltjes-Wigert matrix model, and we can solve it in a variety of ways $[12,13,26]$. The direct computation performed in [13] is the most convenient one in view of the two-cut solution that we will introduce later, so let us briefly review it. As usual, we introduce a resolvent

$$
\omega_{0}(\lambda)=\int d \lambda^{\prime} \frac{\rho\left(\lambda^{\prime}\right)}{\lambda-\lambda^{\prime}},
$$

which due to the normalization (2.10) and the redefinition (3.23), satisfies the following asymptotic behavior

$$
\omega_{0}(\lambda)=\frac{1}{\lambda}+\mathcal{O}\left(\lambda^{-2}\right)
$$

as $\lambda \rightarrow \infty$. The density $\rho(\lambda)$ is recovered from the resolvent $\omega_{0}(\lambda)$ through the standard equation

$$
\rho(\lambda)=-\frac{1}{2 \pi i}\left(\omega_{0}(\lambda+i \epsilon)-\omega_{0}(\lambda-i \epsilon)\right) .
$$

We are looking for a one-cut solution to the problem, therefore we assume that the density of eigenvalues is supported in the interval $\left(a^{-}, a^{+}\right)$, where

$$
a^{ \pm}=e^{ \pm t a+t / p} .
$$

It is well known that $\omega_{0}(\lambda)$ can be computed as [27]

$$
\omega_{0}(\lambda)=r(\lambda) \oint_{C} \frac{d z}{2 \pi i} \frac{g(z)}{(\lambda-z) r(z)},
$$

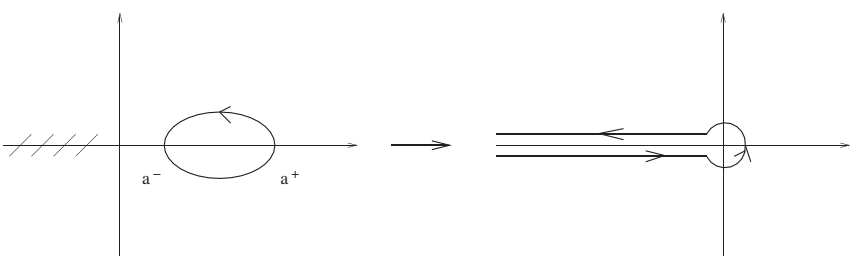

FIG. 2. This figure shows the deformation of the contour needed to compute the resolvent in (3.29). We pick a residue at $z=\lambda$, and we have to encircle the singularity at the origin as well as the branch cut of the logarithm, which on the left hand side is represented by the dashed lines.

where $C$ is a contour around the cut $\left(a^{-}, a^{+}\right)$, and

$$
g(\lambda)=\frac{p}{2 t} \frac{\log \lambda}{\lambda}, \quad r(\lambda)=\sqrt{\left(\lambda-a^{-}\right)\left(\lambda-a^{+}\right)} .
$$

The standard way to compute an integral like (3.31) is to deform the contour. Since the logarithm has a branch cut, we cannot push the contour to infinity. Instead, we deform the contour as indicated in Fig. 2. We pick a pole at $z=\lambda$, and then we surround the cut of the logarithm along the negative real axis and the singularity at $z=0$ with a small circle $C_{\epsilon}$ of radius $\epsilon$. A similar situation appears in, for example, [28]. The final formula for the resolvent is

$$
\begin{aligned}
\omega_{0}(\lambda)= & \frac{p}{2 t} \frac{\log \lambda}{\lambda}-\frac{p}{2 t} r(\lambda) \lim _{\epsilon \rightarrow 0}\left\{-\int_{-\infty}^{-\epsilon} \frac{d z}{z(z-\lambda) r(z)}\right. \\
& \left.+\oint_{C_{\epsilon}} \frac{d z}{2 \pi i} \frac{\log z}{z(z-\lambda) r(z)}\right\} .
\end{aligned}
$$

The integrals in the second line have $\log \epsilon$ singularities as $\epsilon \rightarrow 0$, but they cancel each other, and after some computations one finds for the resolvent:

$$
\begin{aligned}
\omega_{0}(\lambda)= & -\frac{p}{2 t \lambda} \log \left[\frac{\left(\sqrt{a^{-}} \sqrt{\lambda-a^{+}}-\sqrt{a^{+}} \sqrt{\lambda-a^{-}}\right)^{2}}{\left(\sqrt{\lambda-a^{-}}-\sqrt{\lambda-a^{+}}\right)^{2} \lambda^{2}}\right] \\
& +\frac{p}{2 t \lambda} r(\lambda) \frac{1}{\sqrt{a^{-} a^{+}}} \log \left[\frac{4 a^{-} a^{+}}{2 \sqrt{a^{-} a^{+}}+a^{-}+a^{+}}\right] .
\end{aligned}
$$

In order to satisfy the asymptotics (3.26) the second term must vanish, and the first one must go like $1 / \lambda$. This implies

$$
\begin{aligned}
4 a^{-} a^{+} & =2 \sqrt{a^{-} a^{+}}+a^{-}+a^{+}, \\
\sqrt{a^{-}}+\sqrt{a^{+}} & =2 e^{t / p},
\end{aligned}
$$

and from here we obtain the positions of the endpoints of the cut $a^{-}, a^{+}$as a function of $t / p$ :

$$
\begin{aligned}
& a^{-}=2 e^{2 t / p}-e^{t / p}-2 e^{(3 t / 2 p)} \sqrt{e^{t / p}-1}, \\
& a^{+}=2 e^{2 t / p}-e^{t / p}+2 e^{(3 t / 2 p)} \sqrt{e^{t / p}-1} .
\end{aligned}
$$


The final expression for the resolvent is then

$$
\omega_{0}(\lambda)=-\frac{p}{t \lambda} \log \left[\frac{1+e^{-t / p} \lambda+\sqrt{\left(1+e^{-t / p} \lambda\right)^{2}-4 \lambda}}{2 \lambda}\right],
$$

and from here we easily find the density of eigenvalues

$$
\rho(\lambda)=\frac{p}{\pi t \lambda} \tan ^{-1}\left[\frac{\sqrt{4 \lambda-\left(1+e^{-t / p} \lambda\right)^{2}}}{1+e^{-t / p} \lambda}\right] .
$$

We can now go back to the original variable $h$, to find

$$
\rho(h)=\frac{p}{\pi} \tan ^{-1}\left[\frac{\sqrt{e^{A / p^{2}}-\cosh ^{2}(A h /(2 p))}}{\cosh (A h /(2 p))}\right],
$$

which has its support on $(-a, a)$ with

$$
a=\frac{2 p}{A} \cosh ^{-1}\left(e^{A /\left(2 p^{2}\right)}\right) .
$$

As a test of this result, notice that in the double-scaling limit (3.11) one finds

$$
\rho(h)=\rho_{G}(h, 1 / A)+\mathcal{O}\left(1 / p^{2}\right),
$$

therefore the leading term is exactly the Wigner semicircle distribution obtained by [7].

In order to assess the possibility of phase transitions, we have to verify the condition (2.11). Notice first that $\left|\tan ^{-1}(x)\right| \leq \frac{\pi}{2}$, therefore

$$
\rho(h) \leq p / 2
$$

for all $h$. A first conclusion is that there is no phase transition for $p \leq 2$. For $p>2$ there is indeed a phase transition which occurs when the value of $A$ is such that the maximum of the distribution reaches the value 1 . Since the maximum occurs at $h=0$, we immediately find the following line of critical points:

$$
A_{*}(p)=p^{2} \log \left(1+\tan ^{2}\left(\frac{\pi}{p}\right)\right), \quad p>2 .
$$

As $p \rightarrow \infty$,

$$
A_{*}(p) \rightarrow \pi^{2},
$$

in agreement with the result of Douglas and Kazakov (2.4). Notice that $A_{*}(p)$ is a decreasing function of $p$ for $p>2$, and as $p \rightarrow 2^{+}$, the critical area increases to infinity. For a given $p$, the small area phase occurs for $A \leq A_{*}(p)$, and in this phase the planar free energy is well described by the distribution (3.37).

We then have the phase diagram represented in Fig. 3. The horizontal axis represents the parameter $p$, while the vertical axis represents $A$. The critical line, described by the function (3.41), has two asymptotes, represented by dashed lines: as $p \rightarrow \infty$ it approaches the horizontal dashed line $A=\pi^{2}$, which corresponds to the DouglasKazakov phase transition. As $p \rightarrow 2^{+}$it approaches the

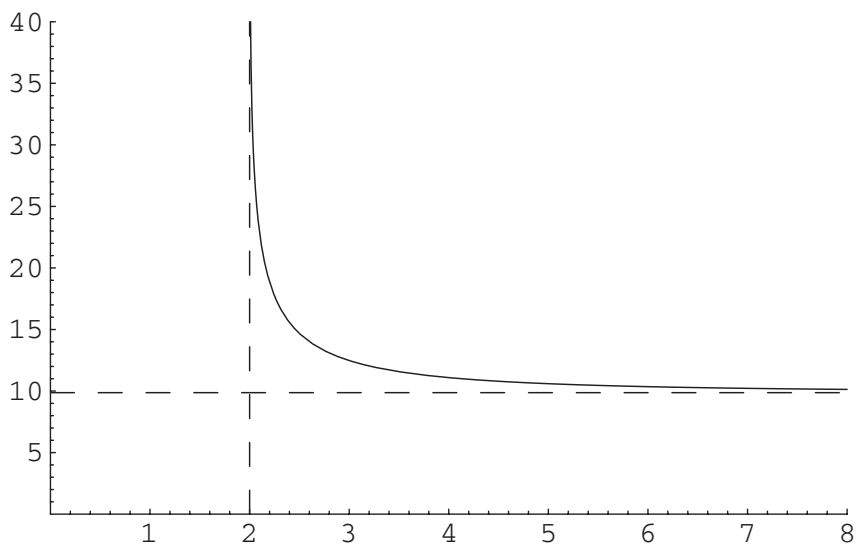

FIG. 3. This figure represents the phase diagram of $q$-deformed 2D YM theory. The horizontal axis represents the parameter $p$, while the vertical axis represents $A$. The curve shown in the figure is the critical line (3.41), which separates the phases of small and large area. The horizontal dashed line, which is the asymptote of the curve as $p \rightarrow \infty$, represents the $A=\pi^{2}$ critical point of Douglas and Kazakov.

vertical asymptote. For $p \in(0,2]$ there is no phase transition. Notice that, if we parametrize the planar $q$-deformed theory in terms of $p$ and $A$, the region $p \rightarrow$ $\infty$ corresponds to a small deformation, while the region $p<2$ corresponds to a large deformation. We then see that, if we start with ordinary 2D YM and we turn on the deformation parameter $1 / p$, the Douglas-Kazakov phase transition persists although the critical area increases. At $p=2$ there is a "barrier" where the critical area becomes infinite. Therefore, when the deformation parameter is large enough, the large $N$ phase transition is smoothed out.

To find the free energy in the small area phase, we have to compute the functional (3.19) evaluated on the density (3.37). Since this functional is closely related to the functional describing the planar Chern-Simons matrix model, we can borrow the results from [13,25]. From [13] it follows that, at large $N$, the matrix integral (3.23) is given by

$$
\exp \left(N^{2} F_{0}(\xi)\right)
$$

with

$$
F_{0}(\xi)=\frac{1}{\xi^{2}} F_{0}^{\mathrm{CS}}(\xi)+\frac{\xi}{12}
$$

Since $\xi=t / p$ in our example, we finally obtain

$$
F_{0}^{q}(t, p)=\frac{1}{t^{2}}\left(p^{2} F_{0}^{\mathrm{CS}}(t / p)-2 F_{0}^{\mathrm{CS}}(t)\right)+\frac{t}{12 p}+\frac{p t}{24} .
$$

As a further check of this expression, notice that, after using the expansion, 


$$
\mathrm{Li}_{3}\left(e^{-t}\right)=\zeta(3)-\frac{\pi^{2}}{6} t+\left(\frac{3}{4}-\frac{1}{2} \log t\right) t^{2}+\mathcal{O}\left(t^{3}\right),
$$

one finds in the double-scaling limit (3.11)

$$
F_{0}^{q}(t, p) \rightarrow \frac{A}{24}-\frac{1}{2} \log A+\frac{3}{4},
$$

which indeed is the free energy of the usual 2D YM theory in the small area phase.

\section{INSTANTON ANALYSIS}

Since $q$-deformed 2D YM theory is a one-parameter deformation of the standard one, we expect the phase transition discovered in the previous section to be triggered by instantons as well. In this section we will verify this by computing the one-instanton suppression factor in the $q$-deformed case. This will also give an intuitive explanation of why the phase transition is absent for $p \leq 2$.

The starting point of the discussion is to write the partition function of the theory in a way that makes manifest the instanton content of the model. Since $q$-deformed 2D YM theory has the same action as standard 2D YM, but differs in the measure [2], we expect the partition function to be expressed in terms of a sum over instantons,

$$
Z^{q}=\sum_{n_{j}} w\left(n_{j}\right) \exp \left(-\frac{2 \pi^{2} N}{A} \sum_{j=1}^{N} n_{j}^{2}\right),
$$

where $n_{j}, j=1, \cdots, N$, are the instanton numbers characterizing a classical solution [8], and $w\left(n_{i}\right)$ is the weight of such a configuration in the semiclassical expansion. In order to compute the weights $w\left(n_{j}\right)$, we follow the technique used by Minahan and Polychronakos [22] in standard 2D YM and perform a Poisson resummation of the original expression (3.1). This can be regarded as a duality transformation which takes us from the large $A$ phase where the expansion (3.1) is valid, to the small area phase where the semiclassical expansion (4.1) is valid. The partition function can then be written as

$$
Z^{q}=C \sum_{n_{j}} F_{2}\left(2 \pi n_{j}\right)
$$

where $F_{2}\left(x_{j}\right)$ is a Fourier transform with respect to the variables $p_{j}=l_{j}-j+1 / 2$ :

$$
\begin{aligned}
F_{2}\left(x_{j}\right)= & \int \prod_{j} d p_{j} e^{-i \sum_{j} x_{j} p_{j}} \prod_{j<k}\left(2 \sinh \frac{t}{N}\left(p_{j}-p_{k}\right)\right)^{2} \\
& \times \exp \left(-\frac{A}{2 N} \sum_{j} p_{j}^{2}\right),
\end{aligned}
$$

and we are setting the $\theta$ angle to zero. This transform can be performed by first computing

$$
\begin{aligned}
F_{1}\left(x_{j}\right)= & \int \prod_{j} d p_{j} e^{-i \sum_{j} x_{j} p_{j}} \prod_{j<k}\left(2 \sinh \frac{t}{N}\left(p_{j}-p_{k}\right)\right) \\
& \times \exp \left(-\frac{A}{2 N} \sum_{j} p_{j}^{2}\right),
\end{aligned}
$$

and then doing a convolution. The integral (4.4) reduces to a Gaussian after using Weyl's denominator formula for a general Lie algebra,

$$
\sum_{w \in \mathcal{W}} \epsilon(w) e^{w(\rho) \cdot u}=\prod_{\alpha>0} 2 \sinh \frac{\alpha \cdot u}{2},
$$

where $\alpha$ are the positive roots, $w \in \mathcal{W}$ are the elements of the Weyl group, and $\epsilon(w)$ is the parity of $w$. We find, up to a multiplicative constant,

$$
F_{1}\left(x_{j}\right)=\exp \left(-\frac{N}{2 A} \sum_{j=1}^{N} x_{j}^{2}\right) \prod_{j<k} 2 \sin \frac{t}{2 A}\left(x_{j}-x_{k}\right),
$$

and using convolution we finally obtain

$$
\begin{aligned}
F_{2}\left(x_{j}\right)= & \exp \left(-\frac{N}{2 A} \sum_{j=1}^{N} x_{j}^{2}\right) \times \int \prod_{j=1}^{N} d y_{j} \\
& \times \prod_{j<k}\left(4 \sin \frac{t}{2 A}\left(x_{j k}+y_{j k}\right) \sin \frac{t}{2 A}\left(x_{j k}-y_{j k}\right)\right) \\
& \times \exp \left(-\frac{N}{2 A} \sum_{j=1}^{N} y_{j}^{2}\right)
\end{aligned}
$$

where we introduced the notation $x_{j k}=x_{j}-x_{k}$. The instanton weight has then the expression

$$
\begin{aligned}
w\left(n_{j}\right)= & \int \prod_{j=1}^{N} d y_{j} \prod_{j<k}\left(4 \sin \frac{t}{2 A}\left(2 \pi n_{j k}+y_{j k}\right)\right. \\
& \left.\times \sin \frac{t}{2 A}\left(2 \pi n_{j k}-y_{j k}\right)\right) \exp \left(-\frac{N}{2 A} \sum_{j=1}^{N} y_{j}^{2}\right),
\end{aligned}
$$

which is a $q$-deformed version of the result in [22] for standard 2D YM.

As it was pointed out in [8], a precise way to evaluate the importance of instanton contributions to the partition function is to compare the contribution of the one-instanton term in the semiclassical expansion (4.1) to the contribution of the perturbative vacuum. The relative weight of these contributions defines a function $\gamma(A, p)$ as follows

$$
\exp \left[-\frac{N}{A} \gamma(A, p)\right]=\exp \left(-N \frac{2 \pi^{2}}{A}\right) \frac{w_{1}}{w_{0}},
$$

where the exponent in the right hand side involves the instanton action for $n_{1}=1, n_{i>1}=0$, and we have denoted

$$
\frac{w_{1}}{w_{0}}=\frac{w(1,0, \cdots, 0)}{w(0, \cdots, 0)}
$$


We call the function in (4.9) the one-instanton suppression factor. Notice that, as long as $\gamma(A, p)$ is different from zero, instantons will be suppressed in the large $N$ limit. The suppression is bigger the larger $\gamma(A, p)$ is. In the remaining of this section, we will compute $\gamma(A, p)$ in the small area phase of $q$-deformed 2D YM, and we will study its properties.

Let us first define the partition function

$$
Z_{N}=\int \prod_{j=1}^{N} d y_{j} \prod_{j<k}\left(2 \sin \frac{t}{2 A}\left(y_{j}-y_{k}\right)\right)^{2} \exp \left(-\frac{N}{2 A} \sum_{j=1}^{N} y_{j}^{2}\right) .
$$

This is very close to the partition function of the ChernSimons matrix model, although it has a sin interaction between eigenvalues instead of a sinh interaction. We can then use the results of the previous section after changing

$$
p \rightarrow-i \frac{p}{A}, \quad A \rightarrow \frac{1}{A},
$$

and doing carefully the analytic continuation of $p$ to the imaginary axis. Equivalently, we can change variables $y=$ $-i A \beta / t$ in (4.11) to obtain the matrix model (3.21) with $\xi=-A / p^{2}$. One can then see from the formulae presented in the last section that the planar limit of (4.11) is controlled by the following density of eigenvalues,

$$
\zeta(y)=\frac{p}{\pi A} \tanh ^{-1}\left[\frac{\sqrt{\cos ^{2}(y /(2 p))-e^{-A / p^{2}}}}{\cos (y /(2 p))}\right],
$$

with endpoints located at

$$
Y=2 p \cos ^{-1}\left(e^{-A /\left(2 p^{2}\right)}\right) .
$$

As $p \rightarrow \infty$, one can easily check that $\zeta(y) \rightarrow \rho_{G}(y, A)$.

We can now evaluate (4.10). Notice first that $w_{0}=Z_{N}$. On the other hand, as in [8], one has

$$
\begin{aligned}
w_{1}= & Z_{N-1} \int_{-\infty}^{\infty} d y_{1} e^{-(N / 2 A) y_{1}^{2}}\left\langle\prod _ { j = 2 } ^ { N } \left( 4 \sin \frac{1}{2 p}\right.\right. \\
& \left.\left.\times\left(2 \pi+\left(y_{j}-y_{1}\right)\right) \sin \frac{1}{2 p}\left(2 \pi-\left(y_{j}-y_{1}\right)\right)\right)\right\rangle_{N-1},
\end{aligned}
$$

where the correlator is computed in the model (4.11) with $N-1$ variables. Since we are interested in the large $N$ behavior of the one-instanton suppression factor, we can compute the different integrals in the saddle-point approximation. This, in particular, means that we can set $y_{1}=0$ inside the correlator in (4.15). We find,

$$
\begin{aligned}
\frac{w_{1}}{w_{0}}= & \left(\frac{2 \pi A}{N}\right)^{1 / 2} \frac{Z_{N-1}}{Z_{N}} \exp \left\{(N-1) \int d y \zeta(y)\right. \\
& \left.\times \log \left(4 \sin \frac{1}{2 p}(2 \pi+y) \sin \frac{1}{2 p}(2 \pi-y)\right)\right\} .
\end{aligned}
$$

We have first to evaluate the quotient $Z_{N-1} / Z_{N}$ in the large $N$ limit. It is easy to see that, at leading order in $N$, this quotient is

$$
\exp \left\{-N\left(2 F_{0}(\xi)+\xi F_{0}^{\prime}(\xi)\right)\right\}
$$

where $F_{0}(\xi)$ is given in (3.44). Here, $\xi=-A / p^{2}$, and after an analytic continuation $\xi \rightarrow-\xi$ we find,

$$
2 F_{0}(\xi)+\xi F_{0}^{\prime}(\xi)=\frac{p^{2}}{A}\left(\operatorname{Li}_{2}\left(e^{-A / p^{2}}\right)-\frac{\pi^{2}}{6}\right),
$$

up to an overall sign $(-1)^{N}$ in $Z_{N-1} / Z_{N}$. Putting everything together, we obtain the following formula for the function $\gamma(A, p)$ defined in (4.9):

$$
\begin{aligned}
\gamma(A, p)= & 2 \pi^{2}+p^{2}\left(\operatorname{Li}_{2}\left(e^{-A / p^{2}}\right)-\frac{\pi^{2}}{6}\right)-A \int d y \zeta(y) \\
& \times \log \left(4 \sin \frac{1}{2 p}(2 \pi+y) \sin \frac{1}{2 p}(2 \pi-y)\right) .
\end{aligned}
$$

The integral in (4.19) can be evaluated analytically. Notice first that in any matrix model one has

$$
\begin{aligned}
F(v) & \equiv \int d \lambda \rho(\lambda) \log (1-\lambda / v) \\
& =\int_{\infty}^{v} d v^{\prime}\left(\omega_{0}\left(v^{\prime}\right)-1 / v^{\prime}\right) .
\end{aligned}
$$

This follows directly from the definition of the resolvent in (3.25). Taking into account the redefinition (4.12), we find that the integral in (4.19) is given by

$$
2 \operatorname{Re} F\left(e^{-A / p^{2}+2 \pi i / p}\right),
$$

where $F(v)$ is obtained as in (4.20), and the relevant resolvent is (3.35). After some work, and using standard identities for the dilogarithm, one finds the following expression:

$$
\begin{aligned}
\gamma(A, p)= & 2 \pi^{2}-p^{2}\left(\operatorname{Li}_{2}\left(e^{-A / p^{2}}\right)+\frac{\pi^{2}}{6}\right) \\
& +2 p^{2} \operatorname{Re} G\left(f_{+}(p, A), f_{-}(p, A)\right),
\end{aligned}
$$

where

$$
\begin{aligned}
G(x, y)= & \frac{1}{2}(\log x)^{2}+\log x \log (1-y)+\operatorname{Li}_{2}(1-x) \\
& +\operatorname{Li}_{2}(y), \\
f_{ \pm}(p, A)= & \exp \left( \pm A /\left(2 p^{2}\right)+i(\varphi-\pi / p)\right), \\
\varphi= & \tan ^{-1}\left(\frac{\sqrt{e^{-A / p^{2}}-\cos ^{2}(\pi / p)}}{\cos (\pi / p)}\right) .
\end{aligned}
$$

In order to understand the properties of the instanton suppression factor, we have studied (analytically and numerically) the properties of (4.22) as a function of $A$ and $p$ 
for $p>2, A \leq A_{*}(p)$. The main results of this analysis are the following:

1. As $p \rightarrow \infty$, the function $\gamma(A, p)$ becomes the function $\gamma_{\mathrm{GM}}(A)$ introduced in (2.17). This is a consistency check of the solution.

2. For any fixed $p>2, \gamma(A, p)$ takes the value $2 \pi^{2}$ at $A=0$ and then it decreases monotonically as the area is increased. At the critical area (3.47) one has

$$
\gamma\left(A_{*}(p), p\right)=0 .
$$

The vanishing of $\gamma(A, p)$ at the critical area can be proved analytically, since at $A=A_{*}(p)$,

$$
f_{ \pm}\left(p, A_{*}(p)\right)=(\cos (\pi / p))^{\mp 1} e^{-i \pi / p} .
$$

For arguments of this form (which are algebraic numbers) the dilogarithm satisfies nontrivial identities [29] that can be easily shown to lead to (4.30).

3. For $p<p^{\prime}$, one has that $\gamma(A, p)>\gamma\left(A, p^{\prime}\right)$ in their common range $A \leq A_{*}\left(p^{\prime}\right)$.

These properties are illustrated in Fig. 4, which shows the function $\gamma(A, p)$ as a function of the area for the values $p=2.1,3, \infty$, from top to bottom. The above properties show that the one-instanton suppression factor in the small area phase decreases as the area grows, until it vanishes at $A_{*}(p)$. Therefore, at the line of critical points found in section 3, the instantons are not suppressed anymore and they become favorable configurations. This shows that the phase transition for the $q$-deformed theory is indeed triggered by instantons, and follows a mechanism similar to the one studied in [8]: for $A>A_{*}(p)$, the entropy of the instantons dominates over their Boltzmann weight. The above analysis also shows that, as $p$ decreases, the instanton suppression factor becomes bigger and bigger, pushing the critical value of the area to ever larger values. This indicates that the smoothing out of the phase transition for $p \leq 2$ is due to the fact that the instantons are suppressed

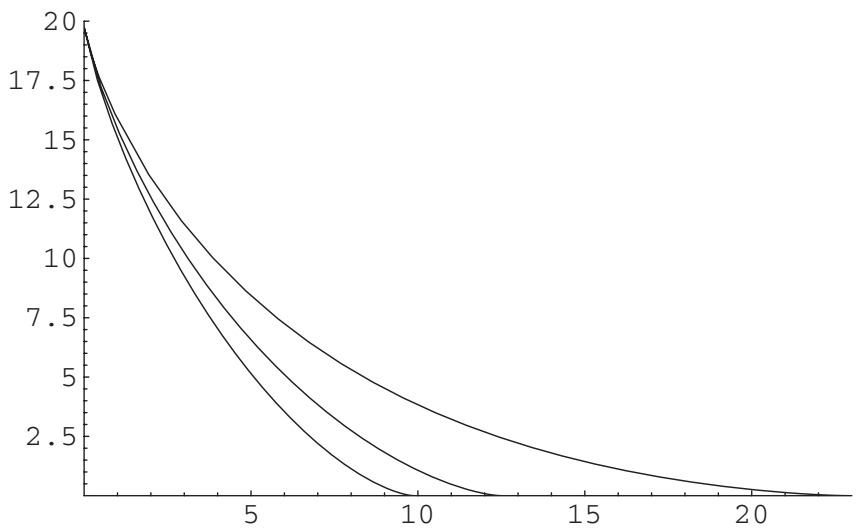

FIG. 4. This figure shows the function $\gamma(A, p)$ appearing in the one-instanton suppression factor, plotted as a function of $A$, and for the values $p=2.1,3, \infty$, from top to bottom. For each $p$ it is a decreasing function of the area and vanishes at the critical value $A_{*}(p)$. for all values of $A$ and we only have one phase dominated by the perturbative vacuum $n_{i}=0$.

\section{THE TWO-CUT SOLUTION}

In this section we give some preliminary results about the large area phase of the theory. After the phase transition found in section 3, we expect a distribution $\rho(h)$ a la Douglas-Kazakov, with the shape shown in the righthand side of Fig. 1 and characterized by two points $\hat{a}, \hat{b}$. The distribution governing the large area distribution is then of the form

$$
\begin{cases}\rho(h)=\tilde{\rho}(h), & -\hat{a} \leq h \leq-\hat{b}, \hat{b} \leq h \leq \hat{a}, \\ 1, & -\hat{b} \leq h \leq \hat{b} .\end{cases}
$$

After changing variables $\lambda=\exp (t h+t / p)$ as in the previous section, the new density of eigenvalues $\tilde{\rho}(\lambda)=$ $1 /(t \lambda) \tilde{\rho}(h)$ has support on the two intervals $\left(a^{-}, b^{-}\right)$, $\left(b^{+}, a^{+}\right)$, where

$$
a^{ \pm}=e^{t / p \pm t \hat{a}}, \quad b^{ \pm}=e^{t / p \pm t \hat{b}} .
$$

This density satisfies the following integral equation,

$$
g(\lambda) \equiv \frac{p}{2 t} \frac{\log \lambda}{\lambda}+\frac{1}{t \lambda} \log \frac{\lambda / b^{+}-1}{\lambda / b^{-}-1}=\mathrm{P} \int \frac{\tilde{\rho}\left(\lambda^{\prime}\right)}{\lambda-\lambda^{\prime}} d \lambda^{\prime} .
$$

As in the one-cut case, we introduce a resolvent

$$
\tilde{\omega}_{0}(\lambda)=\int \frac{\tilde{\rho}\left(\lambda^{\prime}\right)}{\lambda-\lambda^{\prime}} d \lambda^{\prime}
$$

This can be again computed by the contour integral (3.29), but now

$$
r(z)=\sqrt{\left(z-a^{-}\right)\left(z-a^{+}\right)\left(z-b^{-}\right)\left(z-b^{+}\right)},
$$

and $C$ is the union of the contours surrounding the cuts $\left(a^{-}, b^{-}\right),\left(a^{+}, b^{+}\right)$. To perform the integral (3.29) we deform the contours in the way shown in Fig. 5: we now encircle the branch cut along $(-\infty, 0)$, coming from $\log \lambda$, and the branch cut of the integrand along $\left(b^{-}, b^{+}\right)$. The answer for the resolvent is

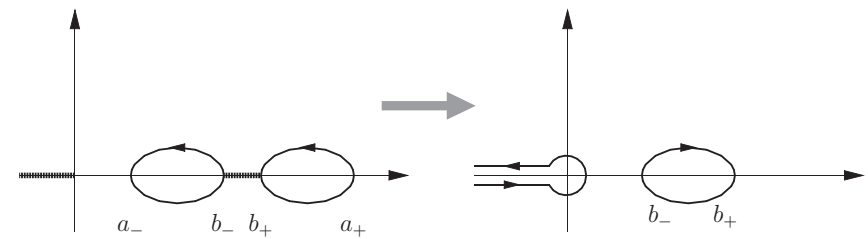

FIG. 5. This figure shows the deformation of the contour needed to compute the resolvent in the two-cut solution. We have to encircle the singularity at the origin, and the two branch cuts denoted by thick lines on the left. 


$$
\begin{aligned}
\tilde{\omega}_{0}(\lambda)= & \frac{p}{2 t} \frac{\log \lambda}{\lambda}+\frac{1}{t \lambda} \log \frac{\lambda / b^{+}-1}{\lambda / b^{-}-1} \\
& -r(\lambda) \frac{p}{2 t} \lim _{\epsilon \rightarrow 0}\left\{-\int_{-\infty}^{-\epsilon} \frac{d z}{z(z-\lambda) r(z)}\right. \\
& \left.+\oint_{C_{\epsilon}} \frac{d z}{2 \pi i} \frac{\log z}{z(z-\lambda) r(z)}\right\} \\
& +\frac{r(\lambda)}{t} \int_{b^{-}}^{b^{+}} \frac{d z}{z(z-\lambda) r(z)} .
\end{aligned}
$$

The above integrals can be expressed in terms of elliptic functions. We will change notation $a^{-}, b^{-}, b^{+}, a^{+}$to $d, c, b, a$. Define

$$
\begin{aligned}
I(\lambda, u) \equiv & \int_{u}^{d} \frac{d z}{(z-\lambda) r(z)} \\
= & \frac{2}{(\lambda-c)(\lambda-d) \sqrt{(a-c)(b-d)}} \\
& \times\{(c-d) \Pi(\phi, n, k)+(d-\lambda) F(\phi, k)\},
\end{aligned}
$$

where $\Pi(\phi, n, k)$ and $F(\phi, k)$ are incomplete elliptic integrals of the third and the first kind, respectively, and

$$
\begin{gathered}
\sin ^{2} \phi=\frac{(a-c)(d-u)}{(a-d)(c-u)}, \quad n=\frac{(a-d)(\lambda-c)}{(a-c)(\lambda-d)}, \\
k^{2}=\frac{(b-c)(a-d)}{(a-c)(b-d)} .
\end{gathered}
$$

In what follows it will be convenient to introduce the following angles $\phi_{1}, \phi_{2}$ and variables $n_{1}$ and $n_{2}$ :

$$
\begin{aligned}
\sin ^{2} \phi_{1} & =\frac{a-c}{a-d}, & \sin ^{2} \phi_{2}=\frac{d}{c} \frac{a-c}{a-d}, \\
n_{1} & =\frac{a-d}{a-c}, & n_{2}=\frac{c}{d} \frac{a-d}{a-c} .
\end{aligned}
$$

In terms of these variables one finds,

$$
\begin{aligned}
I(\lambda,-\infty)= & \frac{2}{(\lambda-c)(\lambda-d) \sqrt{(a-c)(b-d)}} \\
& \times\left\{(c-d) \Pi\left(\phi_{1}, n, k\right)+(d-\lambda) F\left(\phi_{1}, k\right)\right\}, \\
I(\lambda, 0)= & \frac{2}{(\lambda-c)(\lambda-d) \sqrt{(a-c)(b-d)}} \\
& \times\left\{(c-d) \Pi\left(\phi_{2}, n, k\right)+(d-\lambda) F\left(\phi_{2}, k\right)\right\}, \\
I(0,-\infty)= & \frac{2}{c d \sqrt{(a-c)(b-d)}} \\
& \times\left\{(c-d) \Pi\left(\phi_{1}, n_{2}, k\right)+d F\left(\phi_{1}, k\right)\right\} .
\end{aligned}
$$

The first integral in the second line of (5.6) is given by

$$
\frac{1}{\lambda}(I(\lambda,-\infty)-I(\lambda, 0)-I(0,-\infty)+I(0,-\epsilon)) .
$$

The second integral in the second line is simply a residue and it can be computed immediately:

$$
-\frac{1}{\lambda} \frac{1}{\sqrt{a b c d}} \log \epsilon \text {. }
$$

We now compute $I(0,-\epsilon)$ at next-to-leading order in $\epsilon$. This will have a logarithmic singularity which will cancel (5.19). In order to do that, we need the following identity [30]:

$$
\Pi(\phi, n, k)=\delta(n)\left\{\frac{1}{2} \log \frac{\vartheta_{1}(v+\beta)}{\vartheta_{1}(v-\beta)}-\frac{\vartheta_{4}^{\prime}(\beta)}{\vartheta_{4}(\beta)} v\right\},
$$

where

$$
\begin{gathered}
\delta(n)=\left(\frac{n}{(1-n)\left(k^{2}-n\right)}\right)^{1 / 2}, \quad v=\frac{F(\phi, k)}{2 K(k)}, \\
\beta=\frac{F\left(\sin ^{-1}\left(n^{-1 / 2}\right), k\right)}{2 K(k)},
\end{gathered}
$$

and the $\tau$ parameter in the theta functions is given as usual by

$$
q=e^{2 \pi i \tau}=\exp \left(-\pi K^{\prime}(k) / K(k)\right) .
$$

Notice that, when

$$
\sin ^{2} \phi=\frac{1}{n}
$$

we have a logarithmic singularity in the elliptic integral $\Pi(\phi, n, k)$. This is immediately checked in the integral representation of the elliptic function ${ }^{2}$. We can now use (5.13) to extract the next-to-leading behavior. Since

$$
\sin ^{2} \phi=\frac{a-c}{a-d} \frac{d+\epsilon}{c+\epsilon}, \quad n=n_{2},
$$

the leading behavior of $\Pi\left(\phi, k, n_{2}\right)$ is given by

$$
\begin{gathered}
\delta\left(n_{2}\right)\left(-\beta_{2} \frac{\vartheta_{4}^{\prime}\left(\beta_{2}\right)}{\vartheta_{4}\left(\beta_{2}\right)}+\frac{1}{2} \log \frac{\vartheta_{1}\left(2 \beta_{2}\right)}{\vartheta_{1}^{\prime}(0)}-\frac{1}{2} \log \left(\frac{c-d}{4 c d} \delta\left(n_{2}\right)\right)\right. \\
\left.+\frac{1}{2} \log K(k)\right)-\frac{\delta\left(n_{2}\right)}{2} \log \epsilon+\mathcal{O}(\epsilon),
\end{gathered}
$$

where $\beta_{2}$ is given by

$$
\beta_{2}=\frac{F\left(\phi_{2}, k\right)}{2 K(k)} .
$$

This leads to the following expression

$$
I(0,-\epsilon)=-\frac{1}{\sqrt{a b c d}} \log \epsilon+I(0,0)+\mathcal{O}(\epsilon),
$$

where

\footnotetext{
${ }^{2}$ Notice that in the conventions we are using the $n$ in $\Pi(\phi, n, k)$ corresponds to $-n$ in the definition given in [30].
} 


$$
\begin{aligned}
I(0,0)= & \frac{1}{\sqrt{a b c \bar{d}}}\left(-2 \beta_{2} \frac{\vartheta_{4}^{\prime}\left(\beta_{2}\right)}{\vartheta_{4}\left(\beta_{2}\right)}+\log \frac{\vartheta_{1}\left(2 \beta_{2}\right)}{\vartheta_{1}^{\prime}(0)}\right. \\
& \left.+\log \left(\frac{c-d}{4 c d} \delta\left(n_{2}\right)\right)-\log K(k)\right) \\
& +\frac{2}{c \sqrt{(a-c)(b-d)}} F\left(\phi_{2}, k\right) .
\end{aligned}
$$

From the above result we see that the singularities as $\epsilon \rightarrow 0$ cancel, as wished.

We now consider the remaining integral. Define

$$
\begin{aligned}
J(\lambda) \equiv & \int_{c}^{b} \frac{d z}{(z-\lambda) r(z)} \\
= & \frac{2}{(\lambda-a)(\lambda-b) \sqrt{(a-c)(b-d)}} \\
& \times\{(a-b) \Pi(m, k)+(b-\lambda) K(k)\},
\end{aligned}
$$

where

$$
m=\frac{(b-c)(\lambda-a)}{(a-c)(\lambda-b)}
$$

We then have

$$
\int_{c}^{b} \frac{d z}{(z-\lambda) z r(z)}=\frac{1}{\lambda}(J(\lambda)-J(0))
$$

where $J(0)$ is given explicitly as

$$
J(0)=\frac{2}{a b \sqrt{(a-c)(b-\bar{d})}}\{(a-b) \Pi(m(0), k)+b K(k)\} .
$$

Putting everything together, we find the following expression for the resolvent:

$$
\begin{aligned}
\tilde{\omega}_{0}(\lambda)= & \frac{p}{2 t} \frac{\log \lambda}{\lambda}+\frac{1}{t \lambda} \log \frac{\lambda / b-1}{\lambda / c-1} \\
& +\frac{p r(\lambda)}{2 t \lambda}(I(\lambda,-\infty)-I(\lambda, 0)-I(0,-\infty) \\
& +I(0,0))+\frac{r(\lambda)}{t \lambda}(J(\lambda)-J(0))
\end{aligned}
$$

As $\lambda \rightarrow \infty$, this is indeed a Laurent series in $\lambda$ : using again (5.20) it is easy to see that $I(\lambda,-\infty)$ contains a term of the form $-\log (\lambda) / r(\lambda)$ that cancels against the first term in (5.26). In order to derive the conditions for the endpoints of the cut, we must impose the asymptotic behavior

$$
\tilde{\omega}_{0}(\lambda)=\frac{1-2 \hat{b}}{\lambda}+\mathcal{O}\left(\lambda^{-2}\right)
$$

We find three conditions. First of all, notice that there is a term of order $\lambda$ coming from the integrals $I(0,0)$, $I(0,-\infty)$, and $J(0)$. Imposing the cancellation of this term, one obtains the condition

$$
p(I(0,0)-I(0,-\infty))-2 J(0)=0 .
$$

The vanishing of the constant term leads to the condition

$$
p\left(F\left(\phi_{2}, k\right)-F\left(\phi_{1}, k\right)\right)=2 K(k),
$$

Finally, the fact that the $1 / \lambda$ term has the coefficient $1-$ $2 \hat{b}$ leads to a third condition,

$$
\begin{aligned}
& p\left((a+b+d-c)\left(F\left(\phi_{1}, k\right)-F\left(\phi_{2}, k\right)\right)-2(c-d) \Pi\left(\phi_{2}, n_{1}, k\right)\right. \\
&\left.+\sqrt{(a-c)(b-d)}\left(-2 \beta_{1} \frac{\vartheta_{4}^{\prime}\left(\beta_{1}\right)}{\vartheta_{4}\left(\beta_{1}\right)}+\log \frac{\vartheta_{1}\left(2 \beta_{1}\right)}{\vartheta_{1}^{\prime}(0)}-\log \left(\frac{c-d}{4} \delta\left(n_{1}\right)\right)-\log K(k)\right)\right)+2(b+d+c-a) K(k) \\
&++2(d-b) \Pi\left(m_{\infty}, k\right)=t,
\end{aligned}
$$

where

$$
\beta_{2}=\frac{F\left(\phi_{2}, k\right)}{2 K(k)}, \quad m_{\infty}=\frac{b-c}{a-c} .
$$

These conditions determine the endpoints $\hat{a}, \hat{b}$ as functions of the parameters $t, p$. We seem to have three conditions for two unknowns, but since we started with a symmetric problem and we just changed variables, one of the conditions is redundant. This is not easy to verify from the above expressions, but can be checked, for example, by doing a small $t$ expansion of the equations, and assuming a power series ansatz for the endpoints:

$$
\hat{a}(t, A)=\sum_{n=0}^{\infty} \hat{a}_{n}(A) t^{n}, \quad \hat{b}(t, A)=\sum_{n=0}^{\infty} \hat{b}_{n}(A) t^{n} .
$$

The ansatz is justified by the fact that, as $t \rightarrow 0$ with $A$ fixed, we must recover the standard YM result obtained in [7]. One can see that, at leading order in $t$, the three conditions above lead to the same equation, namely

$$
\frac{\hat{a}_{0}+\hat{b}_{0}}{2} A=2 K\left(k_{0}\right)
$$

where

$$
k_{0}^{2}=\frac{4 \hat{a}_{0} \hat{b}_{0}}{\left(\hat{a}_{0}+\hat{b}_{0}\right)^{2}} .
$$

Using standard properties of elliptic functions, one can easily check that this condition becomes 


$$
A=\frac{4}{\hat{a}_{0}} K\left(\hat{b}_{0} / \hat{a}_{0}\right),
$$

which is precisely one of the equations found in [7]. Notice that, by making use of (5.28), we can simplify the expression of the resolvent to

$$
\begin{aligned}
\tilde{\omega}_{0}(\lambda)= & \frac{p}{2 t} \frac{\log \lambda}{\lambda}+\frac{1}{t \lambda} \log \frac{\lambda / b-1}{\lambda / c-1} \\
& +\frac{p r(\lambda)}{2 t \lambda}(I(\lambda,-\infty)-I(\lambda, 0))+\frac{r(\lambda)}{t \lambda} J(\lambda) .
\end{aligned}
$$

In principle, the above conditions for $\hat{a}, \hat{b}$, together with the explicit expression for the resolvent in (5.36), determine completely the solution for the large area phase. These conditions are rather intricate to be treated analytically, but one could study them numerically.

The most important question to address is the order of the phase transition for different values of $p$. This of course can be seen, as in [7], by computing the free energy in the large area phase that we have just analyzed. Since the line of critical points is smoothly connected to the DouglasKazakov transition, we should expect the transition in the $q$-deformed theory to be of third order for any $p>2$. Indeed, one can find indirect evidence that this is the case by using an argument in [8] based on double-scaling limits. If we consider a theory with a large $N n$-th order phase transition at a critical area $A=A_{*}$ between phases I and II, the free energy has the following behavior

$$
F_{0}^{\mathrm{I}}(A)-F_{0}^{\mathrm{II}}(A) \sim\left(A_{*}-A\right)^{n} .
$$

To define a double-scaling limit of such a theory, one should introduce a string coupling constant $\mu_{s}$ through

$$
\mu_{s}^{-2}=N^{2}\left(A_{*}-A\right)^{n} .
$$

The nonperturbative effects of such a theory are expected to be of the form $\exp \left(-1 / \mu_{s}\right)$. But this means that the instanton effects in the original theory should have the behavior $\exp (-N \gamma(A))$, with

$$
\gamma(A) \sim\left(A_{*}-A\right)^{n / 2} .
$$

Indeed, in [8] it is found that the function (2.17) appearing in the instanton suppression factor has exactly the behavior (5.39) with $n=3$ near the Douglas-Kazakov transition point, as required for the existence of a double-scaling limit at a third order phase transition. According to this argument, the behavior of the instanton suppression factor near the critical point can be indeed regarded as an indirect way to probe the order of the phase transition. We have checked numerically that the function $\gamma(A, p)$ that we found in (4.22) behaves indeed as

$$
\gamma(A, p) \sim\left(A_{*}(p)-A\right)^{3 / 2}
$$

near $A_{*}(p)$, for various values of $p>2$. This is indeed consistent with the large $N$ phase transition of the $q$-deformed theory being of third order for all $p>2$.

We should also mention that, in [31], general criteria have been formulated to determine the order of a phase transition for a model based on a distribution of Young tableaux. These criteria only depend on the behavior of the density (3.37) in the small area phase. It can be easily seen that according to these criteria, the phase transition of the $q$-deformed theory is of third order for any $p>2$.

\section{IMPLICATIONS FOR TOPOLOGICAL STRING THEORY AND OPEN PROBLEMS}

In this paper we have shown that $q$-deformed 2D YM theory exhibits an interesting phase structure, with a Douglas-Kazakov phase transition smoothly connected to that of the standard YM theory, and a barrier at $p=2$. One of the original motivations of this analysis was the appearance of the $q$-deformed theory as a nonperturbative completion of topological string theory on certain Calabi-Yau backgrounds. $q$-deformed 2D YM on the sphere has been proposed in [2,3] as a nonperturbative, holographic description of topological strings on the local Calabi-Yau manifold

$$
\mathcal{O}(-p) \oplus \mathcal{O}(p-2) \rightarrow \mathbf{P}^{1},
$$

where the integer number $p>0$ corresponds to the parameter $p$ appearing in (3.1). Explicit computations in [2] show that the perturbative partition function computed in [5] appears as a certain decoupling limit of the large area expansion of the $q$-deformed theory. However, the fact that this theory exhibits a phase transition suggests that, for geometries of the form (6.1) with $p>2$, the large area expansion has a finite radius of convergence which, in terms of the 't Hooft parameter $t$, is given by $t_{*}(p)=$ $A_{*}(p) / p$. As $p$ becomes larger, the radius of convergence becomes smaller. Therefore, the conjecture of [1] suggests that for the geometries (6.1) with $p>2$, there will be a phase transition at small radius in the full, nonperturbatively completed topological string theory. What are the possible interpretations of this phase transition in the topological string theory context? We will mention here three possibilities, although a better understanding of the implications of the phase transition of $q$-deformed YM to nonperturbative topological strings will require a more detailed treatment ${ }^{3}$ :

1. A first possibility is that the phase transition in the $q$-deformed theory indicates a topology change in the Calabi-Yau background. After all, the small and the large area phases are described by different master fields of the two-dimensional theory, corresponding to the one-cut and two-cut solutions discussed above, and it is known that in large $N$ dualities the master field encodes the geometry of

\footnotetext{
${ }^{3}$ The discussion that follows benefited greatly from conversations with M. Aganagic, D. Morrison, H. Ooguri, and N. Saulina.
} 
the target $[12,32]$. This topology change might be also interpreted, as in [6], in terms of a process involving a splitting of baby universes.

2. A second possibility is that the small area phase does not have a geometric interpretation. One indication of that is the string description of standard 2D YM: the analysis of $[4,9]$ shows that the large area expansion has an interpretation in terms of branched coverings of the sphere. However, it has been argued that the existence of a large $N$ phase transition suggests that this geometric picture does not hold for the small area phase [8]. In the same vein, it is likely that the small area phase of the $q$-deformed theory is not described appropriately by topological strings with a geometric target. This is in fact very reminiscent of the analysis of [33] (see also [34,35]), where it was shown that the large $N$ phase transition of the unitary matrix model corresponds, in AdS/CFT at finite temperature, to the point where the horizon of the small AdS black hole becomes comparable to the string scale. At this point, the supergravity/geometry picture breaks down. The situation we are considering here could be a topological string analogue of the large $N$ transition of [33].

3. A more conservative possibility is that the conjecture of [1] does not fully apply to the local geometries (6.1) when $p>2$, or at least does not apply to the small area phase. The original conjecture was formulated for compact Calabi-Yau threefolds, and there may be subtleties when applying it to the noncompact case. It turns out that precisely for $p>2$ there are obstructions for contracting the $\mathbf{P}^{1}$ inside (6.1) to a point [36,37], and because of this reason one can expect these geometries not to arise as a decompactification limit of a compact Calabi-Yau. It is intriguing that the barrier $p=2$ that we found in this paper is the same that occurs in the geometric setting.

In extracting the consequences of our analysis for the nonperturbative physics of topological strings, there is another point that should be mentioned. In our analysis we considered the saddle-point solution of the functional $S[h]$, and we found that this leads to a distribution where $\langle h\rangle=0$ and the dependence on the $\theta$ angle is trivial. However, it has been argued in [16], by studying the instanton weight factors, that the presence of a nonzero $\theta$ changes the location of the critical line. This is an interesting possibility and deserves further study. Also, we have restricted ourselves to solutions with zero $U(1)$ charge. This is indeed the true vacuum of the theory [38], but one could also consider saddle-point solutions like those in [22]: one imposes the constraint $\langle h\rangle=Q$, where $Q$ is the $U(1)$ charge, solves for the density, and then finally sums over all integer charges with a weight $\exp (i Q \theta)$. It may happen that, in order to compare our results with those of [2], one should use this prescription to include the $U(1)$ charges.
It is also worth pointing out that the instanton weight factors considered in section 4 are closely related to the degeneracies of BPS states analyzed in [2]. It is likely that the techniques of [8] that we used and extended to the $q$-deformed case in order to compute these weights lead to a useful technique to obtain the degeneracies.

From the point of view of the two-dimensional gauge theory, the results of this paper indicate that, when the deformation parameter is sufficiently large, the large $N$ phase transition is smoothed out already at the planar level. This is an interesting, new mechanism for smoothing out large $N$ transitions which may have implications in other contexts (the other mechanism we are aware of to smooth out these transitions requires performing a double-scaling limit, as in $[33,39]$, and involves a resummation of the $1 / N$ expansion).

There are also various open questions concerning the gauge theory aspects of our analysis. Of course, the twocut solution that we presented in this paper should be investigated in more detail. One could also investigate the phase structure and free energy of the chiral version of the $q$-deformed theory (in the 2D YM case, this has been done in $[40,41])$. Since the chiral sector makes a more direct contact with the perturbative topological string amplitudes, this may help in understanding better the holographic description proposed in $[2,3]$. It would be also very interesting to analyze the subleading $1 / N$ corrections to the planar result in the small area phase. In [8] this was done for the standard YM case by using a discretized version of orthogonal polynomials, but it is not obvious how to generalize this to a discrete model with a sinh interaction. Such a generalization would make it also possible to define a double-scaled theory near the critical line of the $q$-deformed theory, as we briefly discussed in the last section.

\section{ACKNOWLEDGMENTS}

We would like to thank Mina Aganagic, David Morrison, Andy Neitzke, Hirosi Ooguri, Natalia Saulina, and Erik Verlinde for helpful discussions. M. M. would also like to thank Luis Álvarez-Gaumé and Spenta Wadia for conversations on related topics during the last year. M.M. and A.S. would like to thank the KITP at Santa Barbara, as well as the organizers of the program Mathematical Structure of String Theory, for their hospitality and the great research environment during the completion of this work. While at KITP, their research was supported by the NSF under Grant No. PHY99-07949. The work of X. A., R.B., and A.S. has been partially supported by Stichting FOM. 
[1] H. Ooguri, A. Strominger, and C. Vafa, Phys. Rev. D 70, 106007 (2004).

[2] M. Aganagic, H. Ooguri, N. Saulina, and C. Vafa, Nucl. Phys. B715, 304 (2005).

[3] C. Vafa, hep-th/0406058.

[4] D. J. Gross and W.I. Taylor, Nucl. Phys. B400, 181 (1993).

[5] J. Bryan and R. Pandharipande, math.ag/0411037.

[6] R. Dijkgraaf, R. Gopakumar, H. Ooguri, and C. Vafa, hepth/0504221.

[7] M. R. Douglas and V. A. Kazakov, Phys. Lett. B 319, 219 (1993).

[8] D. J. Gross and A. Matytsin, Nucl. Phys. B429, 50 (1994).

[9] D. J. Gross, Nucl. Phys. B400, 161 (1993).

[10] W. Taylor, hep-th/9404175.

[11] M. Mariño, Commun. Math. Phys. 253, 25 (2005).

[12] M. Aganagic, A. Klemm, M. Mariño, and C. Vafa, J. High Energy Phys. 02 (2004) 010.

[13] M. Mariño, hep-th/0410165.

[14] M. Tierz, Mod. Phys. Lett. A 19, 1365 (2004).

[15] S. de Haro and M. Tierz, hep-th/0501123.

[16] D. Jafferis and J. Marsano, hep-th/0509004.

[17] N. Caporaso, M. Cirafici, L. Griguolo, S. Pasquetti, D. Seminara, and R. J. Szabo, hep-th/0509041.

[18] S. Cordes, G. W. Moore, and S. Ramgoolam, Nucl. Phys. B, Proc. Suppl. 41, 184 (1995).

[19] D. J. Gross and E. Witten, Phys. Rev. D 21, 446 (1980).

[20] S. R. Wadia, Phys. Lett. B 93, 403 (1980).

[21] M. Caselle, A. D'Adda, L. Magnea, and S. Panzeri, hepth/9309107.

[22] J. A. Minahan and A. P. Polychronakos, Nucl. Phys. B422, 172 (1994)
[23] E. Buffenoir and P. Roche, Commun. Math. Phys. 170, 669 (1995).

[24] C. Klimcik, Commun. Math. Phys. 217, 203 (2001).

[25] R. Gopakumar and C. Vafa, Adv. Theor. Math. Phys. 3, 1415 (1999).

[26] N. Halmagyi and V. Yasnov, hep-th/0311117.

[27] N. I. Muskhelishvili, Singular Integral Equations (Dover, New York, 1992).

[28] V. A. Kazakov, M. Staudacher, and T. Wynter, Commun. Math. Phys. 177, 451 (1996).

[29] A. N. Kirillov, hep-th/9212150.

[30] H. Bateman, Higher Transcendental Functions, Vol. 2 (McGraw Hill, New York, 1953).

[31] M. Alimohammadi and M. Khorrami, Nucl. Phys. B597, 652 (2001).

[32] R. Dijkgraaf and C. Vafa, Nucl. Phys. B644, 3 (2002).

[33] L. Álvarez-Gaumé, C. Gómez, H. Liu, and S. Wadia, Phys. Rev. D 71, 124023 (2005).

[34] L. Álvarez-Gaumé, P. Basu, M. Mariño and S. Wadia (to be published).

[35] P. Basu and S. R. Wadia, hep-th/0506203 [Phys. Rev. D (to be published)].

[36] S. Katz and D. Morrison, J. Algebr. Geom.1, 449 (1992).

[37] H. B. Laufer, Ann. Math. Stud. 100, 261 (1981).

[38] A. Aghamohammadi, M. Alimohammadi, and $M$. Khorrami, Mod. Phys. Lett. A 14, 751 (1999).

[39] H. Liu, hep-th/0408001.

[40] M. J. Crescimanno and W. Taylor, Nucl. Phys. B437, 3 (1995).

[41] I. K. Kostov, M. Staudacher, and T. Wynter, Commun. Math. Phys. 191, 283 (1998). 